\title{
小行星探测目标选择与转移轨道方案设计
}

\author{
崔平远 ${ }^{(1)}$ ，乔栋 ${ }^{11}$ ，崔祜涛 ${ }^{(2)}$ 栾恩杰 ${ }^{(3)}$ \\ (1) 北京理工大学宇航学院深空探测技术研究所, 北京 100081 ; \\ (2) 哈尔滨工业大学深空探测基础研究中心, 哈尔滨 150080; \\ (3) 国家国防科技工业局科技委，北京 100048 \\ *E-mail: cuipy@bit.edu.cn \\ 收稿日期: 2009-03-06; 接受日期: 2009-10-23 \\ 国家自然科学基金(批准号: 10832004)资助项目
}

\begin{abstract}
摘要系统地研究了我国开展小行星探测目标选择的技术方法和转移轨道设计方案, 提出 了一套完整的小行星探测任务目标选择与转移轨道方案设计方法. 首先, 针对小行星探测任 务, 讨论分析了具有较大科学探测价值目标星的选择问题; 然后, 针对探测目标的可接近性评 价问题，提出了一种多次借力机制的可接近性评价方法，对探测目标进行䇻选与评估，得到科 学价值与工程可实现性兼备的目标星; 根据探测任务发射时段等约束, 基于 Pork-chop 图法确 定出小行星探测任务的目标星; 最后, 根据探测目标的轨道特性, 提出了一种基于等高线图法 的借力天体选择方法, 给出了 $2: 1 \Delta V$-EGA 探测小行星的转移轨道方案. 通过对该方案的分析, 给出了一种多目标交会转移轨道设计与优化方法, 将原方案扩展为中途可飞越两颗主带小行 星的“一探三”任务方案, 以增加探测任务的科学回报.
\end{abstract}

关键词

小行星探测

目标选择

多目标交会

转移轨道方案
小行星已经成为 21 世纪深空探测活动的重要探 测目标之一. 早在 1991 和 1993 年, 美国的 Galileo 探 测器在探测木星的同时就顺访了 2 颗主带小行星 Gaspra和 Ida, 从而揭开了小行星探测的序幕. 1996 年 2 月, 美国发射了首颗小行星探测器 NEAR, 探测的 目标星选择了 $\mathrm{S}$ 型的 433Eros 小行星,任务途中顺访了 C 型的 253 Mathilde 小行星, 通过对小行星物理、地 质、磁场等的探测, 获得了大量的科学数据. 2003 年 5 月, 日本发射了 Hayabusa 探测器, 探测的目标星选 择了 25143Itokawa 小行星执行采样返回任务, 期望从 采集的样品中得到太阳系及其行星演化的线索. 2007 年 9 月, 美国发射了“黎明(Dawn)”探测器, 以巨型小 行星 Vesta 和矮行星 Ceres 为目标, 探测太阳系早期
形成和演化的条件与进程. 随着小行星探测活动的开 展和探测任务与目标的顺利实现, 进一步推动了深空 探测技术的发展和小行星科学探测与研究的深入.

对于小行星探测任务而言，探测目标的选择无 疑是任务设计与规划的第一步. 与大的行星 (火星、金 星等)探测不同, 小行星为数众多, 目前已发现的约 有 40 多万颗, 正式编号的已经超过 20 万颗, 而且这 个数目还在不断增长. 不同光谱类型和物理特性的 小行星, 对于科学研究有着不同的参考价值, 而不同 的轨道特征, 对于任务的工程实现又有着不同的技 术要求, 如何从为数众多的小行星中选择出科学价 值与工程可实现性兼备的目标星, 是小行星探测任 务设计的一个重要问题. 如果目标星选定, 那么转移

引用格式: Cui P Y, Qiao D, Cui H T, et al. Target selection and transfer trajectories design for exploring asteroid mission. Sci China Tech Sci, 2010, 53: 1150-1158, doi: 10.1007/s11431-010-0007-6 
轨道的方案设计将是任务设计面临的又一个关键问 题, 该问题直接影响到运载工具的选择、探测器的设 计以及对地面测控系统的要求.

本文针对我国首个小行星探测任务的构想方案 所涉及的探测任务目标选择、可接近性评价等问题进 行了系统的研究. 首先, 基于小行星的光谱类型和物 理特性等, 讨论分析了具有较大科学探测价值目标 星的选择问题; 然后, 提出一种多次借力机制的可接 近性评价方法, 对探测目标进行篮选与评估, 并根据 探测任务发射时段等约束, 基于 Pork-chop 图法确定 探测任务的目标星; 最后, 基于能量等高线图法提出 一种借力天体选择方法, 给出 $2: 1 \Delta V$-EGA 探测小行 星的转移轨道方案. 通过对方案的分析, 给出了一种 多目标交会转移轨道设计与优化方法, 将原方案扩 展为中途可飞越两颗小行星的“一探三”任务方案, 以增加探测任务的科学回报. 本文的研究工作较好 地解决了小行星探测任务的目标选择与转移轨道方 案设计问题, 可为我国未来开展的小行星探测任务 的规划与系统方案的设计提供技术参考.

\section{1 小行星探测任务的目标选择}

\section{1 小行星探测任务的科学目标选择}

由于受到技术水平的限制，早期在选择探测目 标时, 主要考虑它的可接近性. 随着技术的进步和深 空探测任务的进一步开展, 科学家们对探测目标的 选择渐渐趋向于目标本身所具有的科学价值. 在科 学目标星选择时主要考虑的是目标星所具有的特殊 物理性质, 这些物理性质可能为太阳系的起源、行星 动力学演化等重要课题的研究提供重要的线索, 这 些物理性质主要包括光谱类型(例如表现出外层主带 小行星原始物质特性的 $\mathrm{C}$ 类; 可能富含金属的 $\mathrm{M}$ 类; 可能是从 Vesta 中分离出来的 V 类; 反照率非常低, 光谱主要在红色, 主要分布在小行星带极外层的 $\mathrm{P}$ 类、可能为彗星研究提供重要研究线索的 D 类等)、 非主轴自旋、超慢自旋、超快自旋、双体结构等(异 常的自旋状态也可能为推测彗星的演化提供重要的 线索, 双体结构可能为小行星动力学的演化提供重 要线索等).

近地小行星(near earth asteroids)是指轨道与地球 轨道相交的小行星群体. 按轨道类型划分, 近地小行 星可以分为三类: 1) Aten 类, 轨道半长轴小于 $1 \mathrm{AU}$,
且远日点日心距 $Q$ 大于地球的近日点日心距(即 $a<1$ $\mathrm{AU}, Q>0.983 \mathrm{AU}) ; 2$ ) Apollo 类, 轨道半长轴大于 1 $\mathrm{AU}$, 且近日点日心距 $q$ 大于地球的远日点日心距(即 $a>1 \mathrm{AU}, q>1.017 \mathrm{AU}) ; 3)$ Amor 类, 轨道半长轴大于 1 $\mathrm{AU}$, 且近日点日心距 $q$ 在 1.017 与 $1.3 \mathrm{AU}$ 之间(即 $a>1 \mathrm{AU}, 1.017 \mathrm{AU}<q<1.3 \mathrm{AU})$.

研究发现在主带小行星中所能找到的具有特殊 物理性质的小行星, 在近地小行星群体中也能找到 其对应的目标. 所以, 探测近地小行星对于了解太阳 系的起源、演变历史和现状, 进一步认识地球环境的 形成与演变过程, 探索生命的起源等具有重要意义. 同时，近地小行星在技术的可实现性上也占据一定 的优势. 因而, 在近地小行星中选择科学价值与工程 可实现性兼备的探测目标具有重要的科学与工程意 义. 当然, 随着人类观测手段的提升和认识的深入, 将会有更多具有独特物理性质的小行星被发现，同 时，原有不明确的近地小行星信息将会被更新，因此， 这里不再总结给出具体的探测目标.

为使被选择的目标星既具有重要的科学探测价 值, 又具有技术上的可实现性, 本文下面探讨探测目 标的可接近性评价方法, 以便从技术可实现性方面 对探测目标进行篮选.

\section{2 基于多次借力的小行星可接近性评价}

可接近性通常是通过实现与目标星的交会任务 所需的总的速度增量的大小来评价其优劣. 全局最 优两脉冲转移的评价方法是进行探测目标可接近性 评价与分析的经典方法 ${ }^{[1 \sim 4]}$, 但该方法在评价那些轨 道半长轴较大或偏心率较大的小行星时通常会失效. 单次借力可以较好的解决该问题 ${ }^{[5]}$, 然而对于少数目 标仍不能满足要求.

针对上述问题，在以前研究的基础上，本节将 提出一种设计简单、技术可行的多次借力星际探测 目标可接近性评价方法. 本方法是通过采用多次地 球借力飞行技术对经典的两脉冲转移策略进行扩展, 从而有效减少交会任务所需的发射能量和总的速度 增量.

基于多次借力的小行星可接近性评价方法可归 结为以下 3 步：1) 全局最优两脉冲转移轨道的求解; 2) 借力飞行轨道类型的选择; 3) 多次借力转移轨道 的拼接与可接近性的评价.

1) 全局最优两脉冲转移 
由异面椭圆轨道最优两脉冲转移的算法 ${ }^{[1,6]}$ 可以 求解得到全局最优两脉冲转移所需总的速度增量的 等高线图. 根据等高线图, 猜测出较好的初值, 通过 迭代算法求解出全局最优两脉冲转移轨道的参数, 然后将地球在两脉冲转移中发射时的平近点角作为 借力策略中地球在借力时的平近点角.

2) 借力飞行轨道类型的选择

多次借力飞行的策略: 探测器从地球发射进入 飞行周期为地球公转周期整数倍, 近日点日心距为 地球平均公转半径的日心大椭圆轨道. 在远日点处 施加一个深空机动, 使得近日点日心距小于地球的 轨道半径, 探测器的轨道和地球的轨道相切或相交, 实现借力飞行. 借力飞行后, 探测器将沿新的日心大 椭圆轨道飞行, 类似在远日点处再次施加一个深空 机动, 使之飞回地球, 进行第二次借力飞行, 依次类 推, 构成多次借力飞行策略. 多次借力策略的示意图, 如图 1 所示.

在多次借力策略中, 一个重要的问题就是借力 轨道类型的选择问题. 为了选择借力轨道的类型, 这 里给出地球借力飞行后的远日点日心距与总的速度 增量之间的关系, 如图 2 所示.

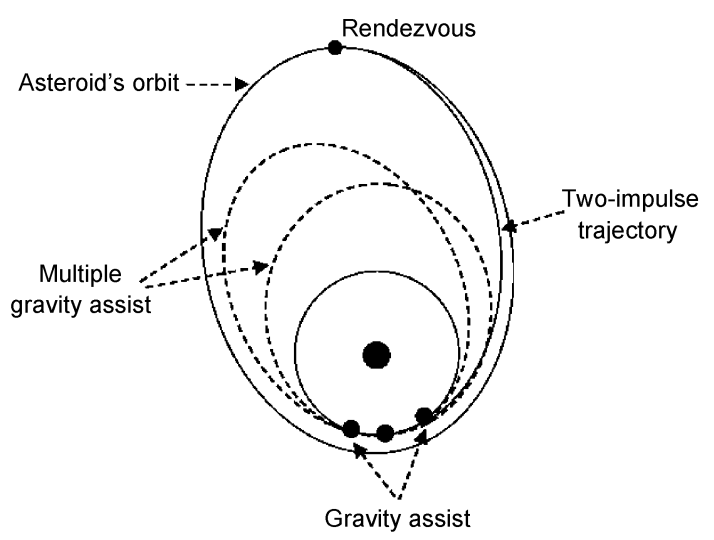

图 1 多次借力可接近性评价策略示意图

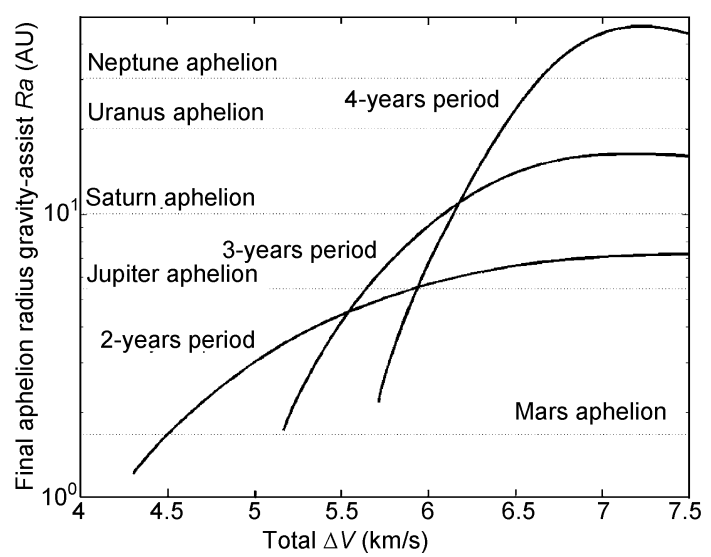

图 $2 \Delta V$-EGA 转移的性能

由图 2 可以看出, 2 年周期的地球借力可以到达 木星附近, 3 年周期的地球借力则远远超过了土星的 远日点. 这是一个重要的性质, 因为小行星和彗星多 数都在这个距离范围内, 因而对于多数小行星和彗 星采用单次 2 年周期的地球借力就可以完成交会; 而 对于少数的目标, 则需采用 3 年周期的地球借力. 由 于 3 年周期地球借力的发射能量 $C_{3}$ 比较大(通常约为 $48 \sim 49 \mathrm{~km}^{2} / \mathrm{s}^{2}$ ), 所以这里通过在 3 年周期地球借力之 前, 再增加一个 2 年周期的地球借力来进一步减少发 射能量和总的速度增量. 这里 2 3 年周期地球借力策 略从发射到最后一次借力的飞行时间约为 5 年, 深空 机动为 2 次.

3) 转移轨道的拼接与可接近性的评价

多次借力飞行策略中借力飞行段是通过 $C_{3}$ 匹配 的方法 ${ }^{[7,8]}$ 得到, 该方法这里不再给出. 这里分别采用 最优两脉冲、 2 年周期地球借力、 3 年周期地球借力和 2 3 年周期地球借力对(6178)1986DA 小行星的可接近 性进行评价. (6178)1986DA小行星是具有较高探测价 值的小天体，但因其具有较大的轨道半长轴(2.5149 $\mathrm{AU})$ 和偏心率(0.5989), 采用传统评价方法无法客观评 估其可接近性. 采用本文评价方法, 结果如表 1 所示.

表 1 采用不同方法的(6178)1986DA 的可接近性评价

\begin{tabular}{ccccccccc}
\hline Profile strategy & $\Delta V_{\text {total }}(\mathrm{km} / \mathrm{s})$ & $\Delta V_{\mathrm{P}}(\mathrm{km} / \mathrm{s})$ & $C_{3}\left(\mathrm{~km}^{2} / \mathrm{s}^{2}\right)$ & $M_{\mathrm{L}}\left({ }^{\circ}\right)$ & $M_{\mathrm{s} 1}\left({ }^{\circ}\right)$ & $M_{\mathrm{s} 2}\left({ }^{\circ}\right)$ & $M_{\mathrm{a}}\left({ }^{\circ}\right)$ & $T_{\mathrm{f}}(\mathrm{d})$ \\
\hline Two-impulse transfer & 7.2353 & 1.3837 & 64.672 & 98.616 & - & - & 303.28 & 1235.70 \\
3-Years Earth gravity-assist & 6.7374 & 1.4826 & 48.828 & 116.856 & 98.616 & - & 303.28 & 2312.92 \\
2-Years Earth gravity-assist & 6.3502 & 2.0012 & 26.424 & 128.80 & 87.663 & - & 53.795 & 937.09 \\
2 3-Years Earth gravity-assist & 6.0796 & 1.7280 & 26.489 & 146.143 & 116.856 & 98.616 & 303.28 & 3013.69 \\
\hline
\end{tabular}


由表 1 可以看出, 与两脉冲转移相比, 采用 2 3 年地球借力策略使得总的速度增量和发射能量分别 减少了 $1.1557 \mathrm{~km} / \mathrm{s}$ 和 $38.183 \mathrm{~km}^{2} / \mathrm{s}^{2}$. 与 3 年地球借力 相比, 2 3 年地球借力策略使得总的速度增量和发射 能量分别减少了 $0.6578 \mathrm{~km} / \mathrm{s}$ 和 $22.339 \mathrm{~km}^{2} / \mathrm{s}^{2}$. 2 3 年 地球借力方案的飞行轨迹如图 3 所示.

采用最优两脉冲和借力机制的可接近性评价方 法, 分别对具有较大科学探测价值 ${ }^{[3]}$ 的近地小行星进 行评价, 其所需总的速度增量(这里主要包括从地球 $200 \mathrm{~km}$ 停泊轨道转移所需速度增量、深空机动及交 会速度增量)小于 $6.0 \mathrm{~km} / \mathrm{s}$, 且飞行时间小于 6 年的目 标星, 如表 2 所示.

表 2 中, $\Delta V_{\text {total }}, \Delta V_{\mathrm{p}}$ 和 $C_{3}$ 分别表示总的速度增量、 发射后的速度增量和发射能量; $M_{\mathrm{L}}, M_{\mathrm{s}}$ 和 $M_{\mathrm{a}}$ 分别表 示发射时地球的平近点角、借力时地球的平近点角和 交会时小行星的平近点角; $T_{\mathrm{f}}$ 表示飞行时间.

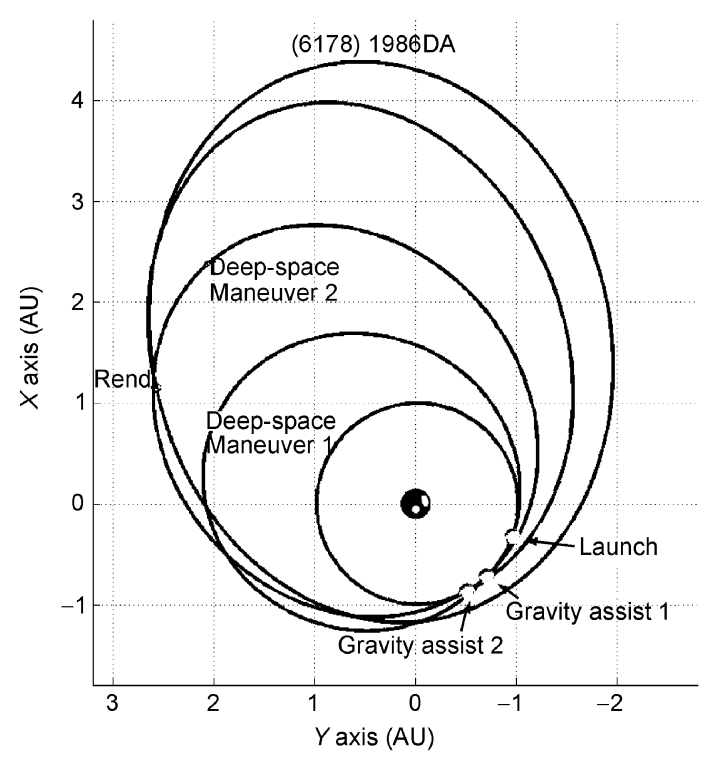

图 3 采用 $2 \sim 3$ 年地球借力策略的飞行轨迹

\section{表 2 探测目标的可接近性评价结果}

\begin{tabular}{|c|c|c|c|c|c|c|c|c|}
\hline & Name & $\Delta V_{\text {total }}(\mathrm{km} / \mathrm{s})$ & $\Delta V_{\mathrm{p}}(\mathrm{km} / \mathrm{s})$ & $C_{3}\left(\mathrm{~km}^{2} / \mathrm{s}^{2}\right)$ & $M_{\mathrm{L}}\left(^{\circ}\right)$ & $M_{\mathrm{S}}\left({ }^{\circ}\right)$ & $M_{\mathrm{a}}\left({ }^{\circ}\right)$ & $T_{\mathrm{f}}(\mathrm{d})$ \\
\hline 1 & $1998 \mathrm{KY} 26$ & 3.92 & 0.18 & 12.05 & 169.2 & - & 224.9 & 338 \\
\hline 2 & 1999SF10 & 3.98 & 0.22 & 12.50 & 295.2 & - & 221.6 & 349 \\
\hline 3 & 2000AE205 & 4.01 & 0.29 & 11.06 & 348.4 & - & 249.0 & 281 \\
\hline 4 & (25143) Itokawa & 4.27 & 0.35 & 16.09 & 144.3 & - & 157.4 & 235 \\
\hline 5 & (10302) 1989ML & 4.28 & 0.52 & 12.07 & 182.5 & - & 172.4 & 243 \\
\hline 6 & (4660) Nereus & 4.44 & 0.23 & 23.09 & 20.0 & - & 114.7 & 206 \\
\hline 7 & (65679) 1989UQ & 4.86 & 1.12 & 11.69 & 261.2 & - & 8.3 & 159 \\
\hline 8 & (35107) $1991 \mathrm{VH}$ & 4.89 & 0.55 & 26.23 & 257.5 & 218.1 & 162.3 & 919 \\
\hline 9 & (7341) 1991VK & 4.97 & 0.66 & 25.34 & 39.2 & 11.7 & 206.05 & 1235 \\
\hline 10 & $1990 \mathrm{OS}$ & 5.00 & 0.67 & 25.92 & 282.2 & 259.0 & 213.3 & 1209 \\
\hline 11 & (33342) 1998WT24 & 5.00 & 0.70 & 25.15 & 21.5 & 340.3 & 346.4 & 773 \\
\hline 12 & $1995 \mathrm{HM}$ & 5.01 & 1.03 & 17.73 & 133.1 & - & 103.1 & 193 \\
\hline 13 & (4179) Toutatis & 5.09 & 0.79 & 25.15 & 338.5 & 297.9 & 247.4 & 1718 \\
\hline 14 & (138911) 2001AE2 & 5.13 & 1.61 & 6.53 & 81.3 & - & 119.8 & 203 \\
\hline 15 & (3288) Seleucus & 5.18 & 0.83 & 26.48 & 142.0 & 111.5 & 230.1 & 1336 \\
\hline 16 & (3908) Nyx & 5.19 & 0.89 & 25.41 & 316.1 & 289.5 & 294.8 & 1454 \\
\hline 17 & (65803) Didymos & 5.22 & 0.92 & 25.43 & 314.4 & 299.5 & 296.5 & 1314 \\
\hline 18 & (98943) 2001CC21 & 5.22 & 1.67 & 7.40 & 152.4 & - & 182.4 & 224 \\
\hline 19 & (3361) Orpheus & 5.28 & 1.22 & 19.45 & 62.5 & - & 143.8 & 180 \\
\hline 20 & (137799) 1999YB & 5.30 & 1.39 & 15.53 & 108.8 & - & 155.9 & 226 \\
\hline 21 & (8034) 1992 LR & 5.34 & 0.99 & 26.49 & 214.0 & 192.5 & 67.0 & 880 \\
\hline 22 & (1627) Ivar & 5.39 & 1.04 & 26.36 & 242.5 & 206.9 & 236.6 & 1258 \\
\hline 23 & (3551) Verenia & 5.40 & 1.08 & 25.65 & 300.3 & 257.6 & 121.7 & 1062 \\
\hline 24 & (6489) Golevka & 5.44 & 1.09 & 26.48 & 217.8 & 176.0 & 53.7 & 906 \\
\hline 25 & (433) Eros & 5.54 & 1.24 & 25.40 & 43.2 & 21.7 & 180.8 & 1006 \\
\hline 26 & (3352) McAuliffe & 5.55 & 1.25 & 25.26 & 32.6 & 12.5 & 126.7 & 1027 \\
\hline 27 & (4015)Wilson-Harrington & 5.63 & 1.32 & 25.63 & 301.3 & 257.2 & 311.3 & 2015 \\
\hline 28 & (887) Alinda & 5.63 & 1.33 & 25.38 & 42.2 & 2.9 & 230.7 & 1563 \\
\hline 29 & (13651) $1997 \mathrm{BR}$ & 5.67 & 1.33 & 26.20 & 260.2 & 195.5 & 212.5 & 960 \\
\hline 30 & (38071) 1999GU3 & 5.89 & 1.54 & 26.46 & 136.5 & 96.7 & 135.3 & 1106 \\
\hline 31 & (31345) 1998 PG & 5.95 & 1.64 & 25.37 & 318.9 & 289.1 & 255.1 & 1328 \\
\hline
\end{tabular}


这里值得注意的是: 可接近性评价方法给出的是平 近点角描述的转移过程, 这一点反映的是轨道之间 的几何关系, 与星历约束无关(星历自由), 对于具体 的探测任务而言, 则需要根据任务的时间约束, 做进 一步的篮选与确定.

\section{3 基于Pork-chop图法的交会机会搜索与探测目 标星的确定}

我国首个小行星探测任务构想给出的主要约束 条件如下: 1) 发射日期在 2010 2015 年;2) 目标小行 星应为已编号的小行星(其轨道信息较为明确); 3) 目 标小行星的远日点日心距小于 $2.7 \mathrm{AU} ; 4)$ 发射后所 需的速度增量(主要包括深空轨道机动和交会时所需 的速度增量)不超过 $1.8 \mathrm{~km} / \mathrm{s} ; 5)$ 所需总的速度增量 不超过 $6.0 \mathrm{~km} / \mathrm{s}$ (主要包括从地球 $200 \mathrm{~km}$ 停泊轨道开 始转移所需的速度增量与发射后速度增量之和). 其 中第 3 项和第 4 项约束与探测器的研制成本和复杂性 紧密相关(尤其与探测器的推进、电源和热控系统有 密切关系), 而第 5 项约束则限制了发射探测器的运 载火箭级别(即应采用小型或中型运载火箭).

按照上述具体的任务约束和要求, 表 2 中目前没 有明确编号的目标、已被其他任务选过的目标、远日点 日心距远远大于 $2.7 \mathrm{AU}$ 的目标、近日点日心距离太阳太 近(例如小于 $0.5 \mathrm{AU})$ 以及重要物理信息缺失的目标, 暂
时不作考虑. 因此, 表 2 中的目标仅有 10 颗基本满足要 求. 基于 Pork-chop 图法 ${ }^{[8]}$ 对这些目标星在 2010 2015 年采用直接转移(DT) 和地球借力转移(EGA) 可能的交 会机会进行分析, 分析表明(13651)1997BR 和(3361) Orpheus 不能满足约束条件, 其余结果如表 3 所示.

由于方案构想将借力飞行技术和多目标交会技术 等作为飞行演示验证的一个重要内容, 所以采用直接交 会的(4660)Nereus 和(10302)1989ML 小行星暂时不作为 备选目标, 同时由表 3 可以看出, (1627)Ivar, (7341)1991VK 和(35107)1991VH 小行星的交会任务在 飞行时间方面更具有优势. 在这 3 颗小行星中, 将 (1627)Ivar 小行星作为主要探测目标, 而将远日点半径 稍大于 $2.7 \mathrm{AU}$ 的(7341)1991VK 和轨道倾角稍大的 (35107)1991VH 作为备选目标星, (4660)Nereus, (10302) 1989ML, (3288)Seleucus, (3908)Nyx 和(8034)Akka 可作 为参考目标. 下面本文将针对(1627)Ivar 的交会任务, 对其转移轨道方案进行设计.

\section{2 小行星探测任务转移轨道方案设计}

\section{1 小行星探测交会任务转移轨道的方案设计}

1) 地球借力 (EGA)转移方案

这里采用 $2: 1 \Delta V$-EGA 技术 ${ }^{[9]}$ 设计转移轨道, 飞 行轨迹如图 4 所示.

\section{表 3 篮选后剩余目标及交会机会}

\begin{tabular}{ccccccc}
\hline Name & $T_{\mathrm{L}}$ (year/month/day) & $T_{\mathrm{f}}(\mathrm{d})$ & $\Delta V_{\text {total }}(\mathrm{km} / \mathrm{s})$ & $\Delta V_{\mathrm{p}}(\mathrm{km} / \mathrm{s})$ & $C_{3}\left(\mathrm{~km}^{2} / \mathrm{s}^{2}\right)$ & Transfer type \\
\hline (1627)Ivar & $2011 / 09 / 20$ & 1064 & 5.36 & 1.00 & 26.2 & EGA \\
& $2011 / 09 / 15$ & 1249 & 5.44 & 1.08 & 26.4 & EGA \\
(3288)Seleucus & $2012 / 05 / 28$ & 1400 & 5.23 & 0.86 & 26.5 & EGA \\
(3908)Nyx & $2013 / 11 / 30$ & 1446 & 5.38 & 1.06 & 25.2 & EGA \\
& $2010 / 11 / 18$ & 1496 & 5.41 & 1.08 & 25.5 & EGA \\
& $2013 / 11 / 16$ & 1687 & 5.47 & 1.14 & 25.6 & EGA \\
& $2010 / 11 / 24$ & 1849 & 5.54 & 1.21 & 25.5 & EGA \\
(4660)Nereus & $2011 / 01 / 11$ & 580 & 4.70 & 0.47 & 23.4 & DT \\
& $2011 / 01 / 09$ & 798 & 4.80 & 0.49 & 25.2 & DT \\
(7341)1991VK & $2013 / 01 / 13$ & 528 & 5.11 & 1.08 & 18.3 & DT \\
(8034)Akka & $2015 / 02 / 14$ & 530 & 5.35 & 1.05 & 24.9 & DT \\
(10302)1989ML & $2010 / 02 / 12$ & 1234 & 5.10 & 0.78 & 25.2 & EGA \\
& $2010 / 02 / 14$ & 1002 & 5.12 & 0.80 & 25.2 & EGA \\
(35107)1991VH & $2010 / 08 / 17$ & 1385 & 5.40 & 1.03 & 26.5 & EGA \\
\hline
\end{tabular}




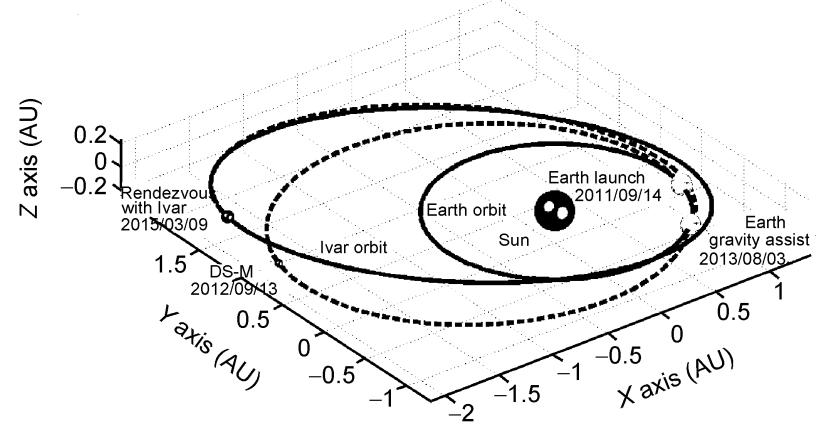

图 4 采用 2:1 $\Delta V$-EGA 方案探测(1627)Ivar 小行星的飞行轨迹

本方案中, 探测器于 2011 年 9 月 14 日从地球 $200 \mathrm{~km}$ 停泊轨道逃逸开始星际航行 (发射能量 $C_{3}$ 为 $26.41 \mathrm{~km}^{2} / \mathrm{s}^{2}$ ), 2012 年 9 月 13 日进行一次深空机动 (大 小为 $0.352 \mathrm{~km} / \mathrm{s}$ ), 2013 年 8 月 3 日进行地球借力 (借力 飞行高度为 $1009.5 \mathrm{~km}), 2015$ 年 3 月 9 日与 Ivar 小行 星交会(交会时所需的速度增量为 $0.76 \mathrm{~km} / \mathrm{s}$ ). 该任务 所需总的速度增量为 $5.477 \mathrm{~km} / \mathrm{s}$, 发射后所需的速度 增量为 $1.112 \mathrm{~km} / \mathrm{s}$, 均满足任务设计要求.

2) 其他行星借力的可行性分析

根据(1627)Ivar 小行星的轨道特性, 如果采用借 力飞行的转移方案, 那么可能的借力天体有三个即 地球、金星和火星. 如何选择借力天体是轨道方案设 计中的一个重要问题. 针对该问题, 本节提出了一种 基于等高线图的借力天体选择方法.

该方法归结起来可以分为以下三步：1) 结合星 历，求解从假设的借力天体到小行星的 Lambert 问题; 2) 根据求解的 Lambert 问题, 给出与小行星交会时 所需速度增量的等高线图(等高线图的绘制可参见文 献[10], 这里不再给出)；3）根据等高线图和任务约 束, 分析该借力天体是否可以作为交会前的最后一 个天体或唯一的借力天体.

基于以上方法，下面讨论金星和火星借力飞行 转移方案的可行性. 从金星和火星借力飞行后与小 行星交会时所需的速度增量等高线图, 如图 5 和图 6 所示.

由图 5 可以看出, 金星作为任务末端借力天体与 小行星交会时所需的速度增量较小的区域有两个, 分别为 2011 年 3 4 月和 2013 年 9 10 月, 交会时所 需的速度增量最小约为 $3.0 \mathrm{~km} / \mathrm{s}$, 不能满足发射后速 度增量小于 $1.8 \mathrm{~km} / \mathrm{s}$ 的约束条件.
由图 6 可以看出, 采用火星作为任务末端借力天 体转移方案的机会相对较多，在 2010 2015 年约有 6 次机会, 较好的一次机会在 2013 年 1 月, 该次机会与 (1627)Ivar 交会时所需速度增量小于 $2.0 \mathrm{~km} / \mathrm{s}$, 优化 后可得交会时最小的速度增量为 $1.39 \mathrm{~km} / \mathrm{s}$, 交会的 日期为 2015 年 8 月 13 日，火星借力的日期为 2013 年 1 月 12 日, 采用圆雉曲线拼接法可以得到如下 4 个火星借力的转移方案, 如表 4 所示.

由表 4 可以看出： 4 种借力方案所需总的速度增

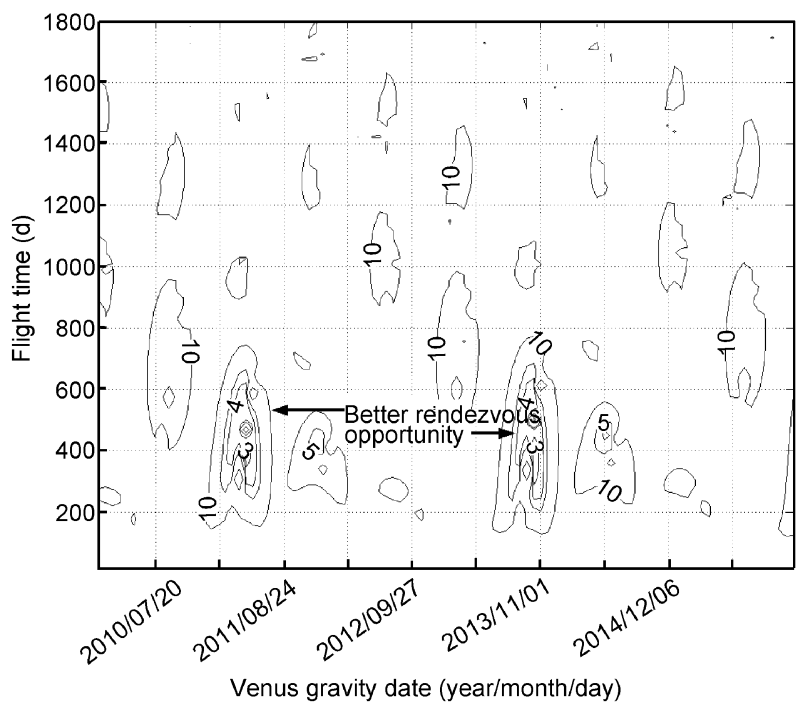

图 5 金星借力探测 Ivar 小行星的机会

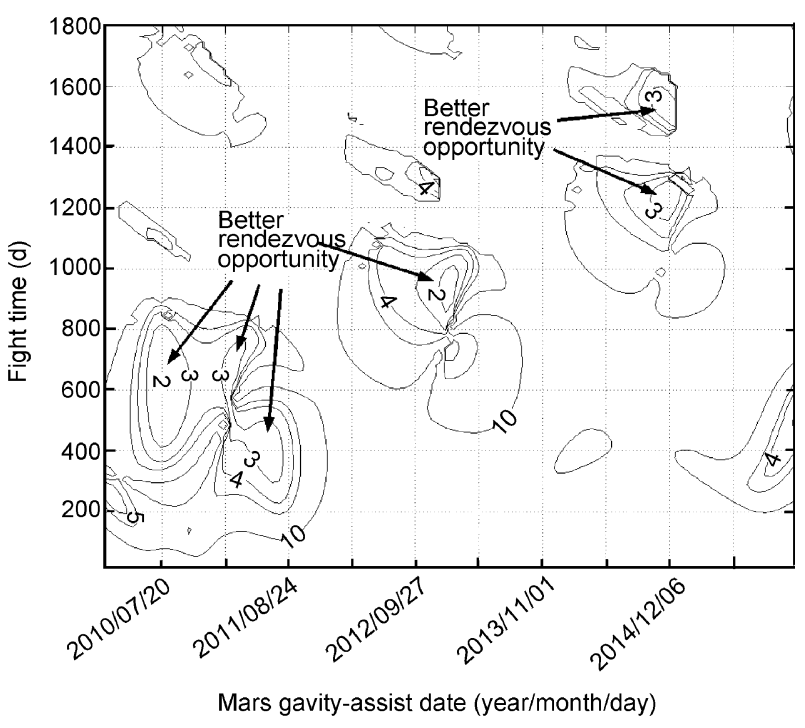

图 6 火星借力探测 Ivar 小行星的机会 
表 4 2010 2015 年火星借力探测 1627Ivar 小行星的可能机会

\begin{tabular}{cccccc}
\hline Profile & $T_{\mathrm{L}}(\mathrm{y}$ ear/month/day $)$ & $T_{\mathrm{f}}(\mathrm{d})$ & $\Delta V_{\text {total }}(\mathrm{km} / \mathrm{s})$ & $C_{3}\left(\mathrm{~km}^{2} / \mathrm{s}^{2}\right)$ & $\Delta V_{\mathrm{r}}(\mathrm{km} / \mathrm{s})$ \\
\hline MGA-1 & $2011 / 10 / 19$ & 1393.8 & 7.41 & 69.5 & 1.39 \\
MGA-2 & $2012 / 02 / 09$ & 1281.0 & 7.55 & 73.3 & 1.39 \\
MGA-3 & $2012 / 02 / 28$ & 1261.9 & 7.88 & 82.4 & 1.39 \\
MGA-4 & $2012 / 03 / 16$ & 1244.5 & 8.07 & 88.2 & 1.39 \\
\hline
\end{tabular}

量均大于 $7.0 \mathrm{~km} / \mathrm{s}$, 且发射能量大于 $60.0 \mathrm{~km}^{2} / \mathrm{s}^{2}$ 不能 满足任务约束的要求.

由以上的设计分析可知, 2:1 $\Delta V$-EGA 转移方案满 足任务设计的所有约束条件. 与两脉冲转移相比, 采用 $2: 1 \Delta V$-EGA 方案后, 发射能量减少了 $23.96 \mathrm{~km}^{2} / \mathrm{s}^{2}$; 总 的速度增量减少了 $0.812 \mathrm{~km} / \mathrm{s}$, 代价是发射后的速度 增量增加了 $0.136 \mathrm{~km} / \mathrm{s}$, 飞行时间增加了 684.6 天.

\subsection{Ivar 小行星探测任务多目标交会轨道方案的 优化}

通过对采用 $2: 1 \Delta V$-EGA 转移方案的飞行轨迹分 析发现，在该任务的飞行过程中可能飞越(4148) McCartney和(358)Apollonia 两颗主带小行星. 针对该 问题, 本文给出一种多目标交会转移轨道设计与优 化方法. 该方法通过 Pork-Chop 图确定设计参数的可 行域, 基于主矢量和变分原理推导出了指标函数与 自由变量的偏导数关系, 最后通过梯度下降法等寻 找最优设计参数.

这里假设探测器从地球发射, 飞越 $n$ 个借力天体 后, 与目标星交会; 在飞越第 $i$ 个借力天体时, 若 $C_{3}$ 不能完全匹配, 则增加深空机动补偿, 那么该任务的 目标函数 $J$ (即总的速度增量) 可以定义为

$$
\begin{aligned}
J= & \left(\sqrt{\left\|\boldsymbol{V}_{\mathrm{L}}-\boldsymbol{V}_{\mathrm{EL}}\right\|^{2}+V_{\mathrm{e} \infty}^{2}}-V_{\mathrm{Lp}}\right) \\
& +\left(\sqrt{\left\|\boldsymbol{V}_{\mathrm{a}}-\boldsymbol{V}_{\mathrm{A}}\right\|^{2}+V_{\mathrm{a} \infty}^{2}}-V_{\mathrm{ap}}\right) \\
& +\sum_{i=1}^{k}\left\|\boldsymbol{V}_{m i+}-\boldsymbol{V}_{m i-}\right\|,
\end{aligned}
$$

这里 $V_{\mathrm{L}}$ 为探测器日心引力场的出发速度, $V_{\mathrm{EL}}$ 为地球 的公转速度, $V_{\mathrm{ex}}$ 为逃逸地球引力场所需的速度, $V_{\mathrm{Lp}}$ 为探测器在停泊轨道上运行的速度; $\boldsymbol{V}_{\mathrm{a}}$ 为探测器日心 引力场的交会速度, $V_{\mathrm{A}}$ 为目标星的公转速度, $V_{\mathrm{a} \infty}$ 为 逃逸目标星引力场所需的速度, $V_{\text {ap }}$ 为探测器在目标
星绕飞轨道上运行的速度; $\boldsymbol{V}_{m i-}$ 和 $\boldsymbol{V}_{m i+}$ 分别为第 $\boldsymbol{i}$ 次 深空机动前后的日心速度.

目标函数 $J$ 相对于发射时间 $t_{\mathrm{L}}$ 的偏导数为

$$
\frac{\partial J}{\partial t_{\mathrm{L}}}=-\left(\Delta \overline{\boldsymbol{V}}_{\mathrm{L}}^{\mathrm{T}} \boldsymbol{F}_{1}+\Delta \overline{\boldsymbol{V}}_{12}^{\mathrm{T}} \boldsymbol{H}_{1}\right)\left(\boldsymbol{V}_{\mathrm{L}}-\boldsymbol{V}_{\mathrm{EL}}\right),
$$

这里 $\Delta \overline{\boldsymbol{V}}_{\mathrm{L}}^{\mathrm{T}}=\left(\boldsymbol{V}_{\mathrm{L}}-\boldsymbol{V}_{\mathrm{EL}}\right) / \sqrt{\left\|\boldsymbol{V}_{\mathrm{L}}-\boldsymbol{V}_{\mathrm{EL}}\right\|^{2}+V_{\mathrm{ex}}^{2}}, \quad \Delta \overline{\boldsymbol{V}}_{12}^{\mathrm{T}}=\left(\boldsymbol{V}_{12}\right.$ $\left.-V_{21}\right) / \sqrt{\left\|V_{21}-V_{12}\right\|^{2}}$, 其中第一个下角标表示轨道段 的标记(例如 1 表示第一段轨道), 第二个下角标表示 轨道的端点(1表示轨道的始端, 2 表示轨道的 末端).

假设转移轨道可以分为 $n$ 段, 则目标函数 $J$ 相对 于到达时间 $t_{\mathrm{a}}$ 的偏导数为

$$
\frac{\partial J}{\partial t_{\mathrm{a}}}=\left(\Delta \overline{\boldsymbol{V}}_{n 1}^{\mathrm{T}} \boldsymbol{E}_{n}+\Delta \overline{\boldsymbol{V}}_{\mathrm{a}}^{\mathrm{T}} \boldsymbol{G}_{n}\right)\left(\boldsymbol{V}_{\mathrm{A}}-\boldsymbol{V}_{\mathrm{a}}\right),
$$

这里 $\Delta \overline{\boldsymbol{V}}_{\mathrm{a}}^{\mathrm{T}}=\left(\boldsymbol{V}_{\mathrm{a}}-\boldsymbol{V}_{\mathrm{A}}\right) / \sqrt{\left\|\boldsymbol{V}_{\mathrm{a}}-\boldsymbol{V}_{\mathrm{A}}\right\|^{2}+V_{\mathrm{a} \infty}^{2}}$, 若最后一段 轨道的始端点为第 $i$ 次深空机动后, 则 $\Delta \overline{\boldsymbol{V}}_{n 1}^{\mathrm{T}}=$ $\left(\boldsymbol{V}_{m i+}-\boldsymbol{V}_{m i-}\right) / \sqrt{\left\|\boldsymbol{V}_{m i+}-\boldsymbol{V}_{m i-}\right\|^{2}}$, 若最后一段轨道的始端点 为第 $i$ 次借力飞行后, 则

$$
\Delta \overline{\boldsymbol{V}}_{n 1}^{\mathrm{T}}=\left(\boldsymbol{V}_{\text {si+ }}-\boldsymbol{V}_{\mathrm{PS}}\right) / \sqrt{\left\|\boldsymbol{V}_{s i+}-\boldsymbol{V}_{\mathrm{PS}}\right\|^{2}} .
$$

假设第 $i$ 次深空机动前的轨道段为 $k$, 深空机动 后的轨道段为 $k+1$, 则目标函数 $J$ 相对于第 $i$ 次深空 机动时间 $t_{m i}$ 的偏导数为

$$
\begin{aligned}
\frac{\partial J}{\partial t_{m i}}= & \left(\Delta \overline{\boldsymbol{V}}_{(k+1) 2}^{\mathrm{T}} \boldsymbol{E}_{k+1}+\Delta \overline{\boldsymbol{V}}_{m i+}^{\mathrm{T}} \boldsymbol{F}_{k+1}\right) \boldsymbol{V}_{m i+} \\
& -\left(\Delta \overline{\boldsymbol{V}}_{k 1}^{\mathrm{T}} \boldsymbol{E}_{k}+\Delta \overline{\boldsymbol{V}}_{m i-}^{\mathrm{T}} \boldsymbol{G}_{k}\right) \boldsymbol{V}_{m i-} .
\end{aligned}
$$

同理, 目标函数 $J$ 相对于第 $i$ 次深空机动位置 $\boldsymbol{R}_{m i}$ 的偏导数为 


$$
\begin{aligned}
\frac{\partial J}{\partial \boldsymbol{R}_{m i}}= & \Delta \overline{\boldsymbol{V}}_{k 1}^{\mathrm{T}} \boldsymbol{E}_{k}+\Delta \overline{\boldsymbol{V}}_{m i-}^{\mathrm{T}} \boldsymbol{G}_{k} \\
& +\Delta \overline{\boldsymbol{V}}_{m i+}^{\mathrm{T}} \boldsymbol{F}_{k+1}+\Delta \overline{\boldsymbol{V}}_{(k+1)^{2}}^{\mathrm{T}} \boldsymbol{H}_{k+1} .
\end{aligned}
$$

在优化过程中借力飞行的时间不作为自由变量, 而是作为满足借力飞行匹配条件的变量. 以上函数 的推导这里不再给出, 可以参见相关文献 ${ }^{[11,12]}$. 对 (1627)Ivar的“一探三”任务进行优化设计后, 其飞行 轨迹如图 7 所示.

由图 7 可以看出, 探测器于 2011 年 9 月 14 日从 地球 $200 \mathrm{~km}$ 停泊轨道逃逸开始星际航行(发射能量 $C_{3}$ 为 $\left.26.69 \mathrm{~km}^{2} / \mathrm{s}^{2}\right), 2012$ 年 6 月 4 日飞越(4148) McCartney 小行星, 2012 年 6 月 5 日进行第一次深空 机动(深空机动大小为 $0.364 \mathrm{~km} / \mathrm{s}$ )；2013 年 8 月 3 日 进行地球借力 (借力时的飞越高度为 $1376.8 \mathrm{~km}$ ); 借 力飞行后, 于 2014 年 7 月 14 日飞越(358)Apollonia 小行星, 2014 年 7 月 24 日进行第二次深空机动(深空 机动的大小为 $0.172 \mathrm{~m} / \mathrm{s}) ; 2015$ 年 3 月 9 日与(1627) Ivar 小行星进行交会(交会时所需的速度增量为 0.572 $\mathrm{km} / \mathrm{s}$ ). 该任务所需总的速度增量为 $5.486 \mathrm{~km} / \mathrm{s}$, 发射 后所需的速度增量为 $1.109 \mathrm{~km} / \mathrm{s}$, 其中包括两次深空 机动和与 Ivar 交会时所需的速度增量. 这里值得一提 的是发射能量和总的速度增量仅增加了 $0.28 \mathrm{~km}^{2} / \mathrm{s}^{2}$ 和 $9 \mathrm{~m} / \mathrm{s}$, 就可以实现途中飞越 2 颗小天体的任务, 从 而提高了该任务的科学回报. 以上给出的是小天体 探测转移轨道的初始方案, 适用于任务的规划与总 体设计, 而对于实际飞行轨道, 则需要在精确轨道动
力学模型下进行进一步的分析计算, 这里不再给出.

\section{3 结论}

针对我国首个小天体探测任务的构想方案所涉 及的目标选择、可接近性评价等问题进行了系统的研 究, 获得了一套完整的小行星探测目标选择与转移 轨道方案设计的方法, 并基于该方法提出了(1627)Ivar 小行星的“一探三”探测方案. 首先, 分析了具有较大 科学探测价值目标选择所考虑的主要因素; 然后, 针 对传统方法评价可接近性可能失效的问题，引入借 力机制，提出了一种多次借力机制的可接近性评价 方法, 对具有较大科学探测价值的近地小行星进行 篎选与评估, 得到 31 颗科学价值与工程可实现性兼 备的目标星. 根据探测任务的约束, 采用 Pork-chop 图法和借力飞行技术对目标星进行了进一步的分析, 确定(1627)Ivar 为 2010 2015 年小天体探测任务的目 标星, (7341)1991VK 等 7 颗为备选目标星; 最后, 针 对借力飞行方案，提出了一种基于等高线图法的借 力天体选择方法，在对金星等借力方案评估的基础 上, 采用 2:1 $\Delta V$-EGA 方法设计了探测(1627)Ivar 小行 星的转移轨道, 分析其飞行轨迹发现途中可能飞越 (4148)McCartney和(358)Apollonia 两颗主带小行星. 针对该问题, 给出了一种多目标交会转移轨道设计 与优化方法，对该方案进行了扩展设计，给出了“一 探三”任务转移轨道方案, 可增加小行星探测任务的 科学回报.
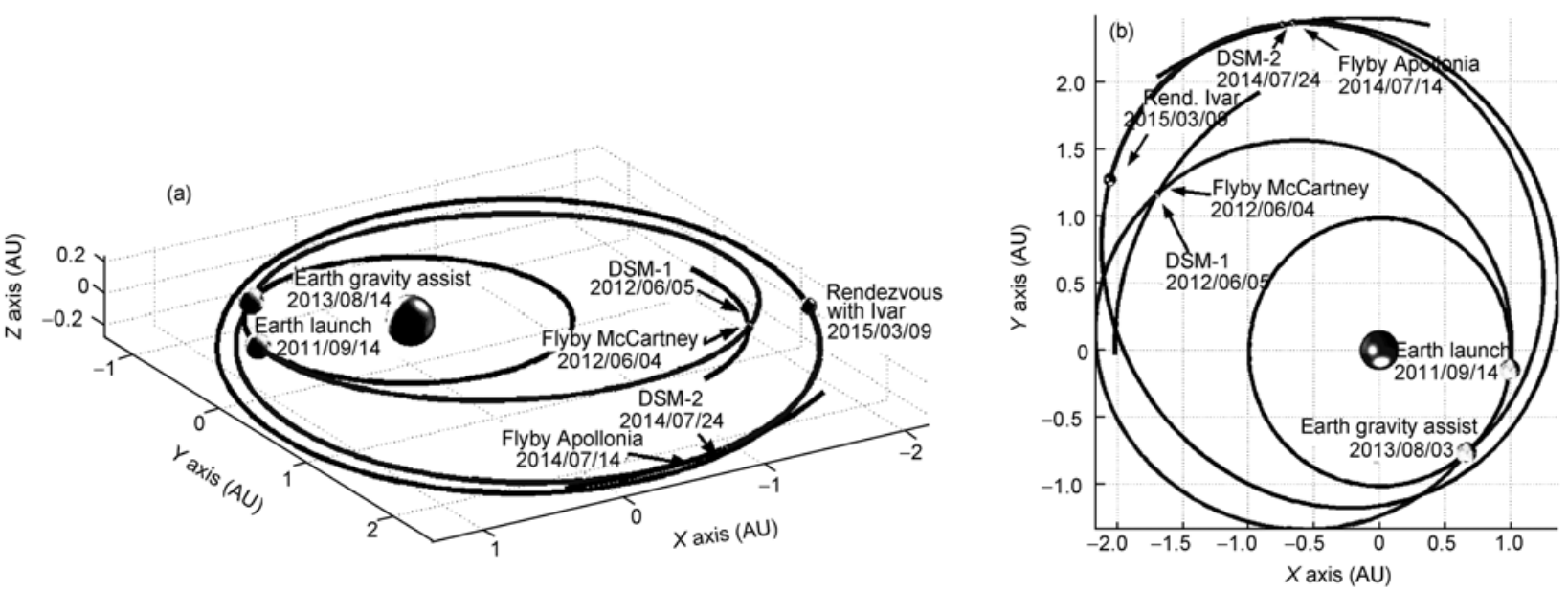

图 7 采用 2:1 $\Delta V$-EGA 方案探测(1627)Ivar 小行星的多次飞越任务的飞行轨迹 


\section{参考文献}

1 Hulkower N D, Lau C O, Bender D F. Optimum two-impulse transfer for preliminary interplanetary trajectory design. J Guid Control Dyn, 1984, 7: 458-461

2 Lau C O, Hulkower N D. Accessibility of Near-Earth asteroids. J Guid Control Dyn, 1987, 10: 225-232

3 Perozzi E, Rossi A, Valsecchi G B. Basic targeting strategies for rendezvous and flyby missions to the Near-Earth asteroids. Planet Space Sci, 2001, 49: 3-22

4 Binzel R P, Perozzi E, Rivkin A S, et al. Dynamical and compositional assessment of Near-Earth object mission targets. Meteoritics Planet Sci, 2004, 39: 351-366

5 Qiao D, Cui H T, Cui P Y. Evaluating accessibility of Near-Earth asteroids via Earth gravity assists. J Guid, Control Dyn, 2006, 29: 502505

6 乔栋, 崔祜涛, 崔平远. 小行星探测最优两脉冲交会轨道设计与分析. 宇航学报, 2005, 26: 362-367

7 Longuski J M, Williams S N. Automated design of gravity-assist trajectories to Mars and the outer planets. Celes Mech Dyn Astron, 1991, 52: 207-220

8 Bonfiglio E P, Longuski J M. Automated design of aerogravity-assist trajectories. J Spac Rock, 2000, 37: 768-775

9 Sims J A, Longuski J M, Staugler A J. $V_{\infty}$ Leveraging for inter planetary missions: multiple-revolution orbit techniques. J Guid Control Dyn, 1997, 20: 409-415

10 郭海林, 汪中生, 曲广吉. 火星探测飞行原理和发射时机分析. 中国科学 E 辑: 技术科学, 2009, 39: 535-543

11 Lorenzo C, Guido C, Dario P. Optimization procedure for preliminary design of opposition-class Mars mission. J Guid Control Dyn, 1998, 21: $134-140$

12 Qiao D, Cui H T, Cui P Y. The design of transfer trajectory for Ivar asteroid exploration. Acta Astronaut, 2009, 65: 1553-1560 